\title{
Evaluation of the hydro-meteorological chain in Piemonte Region, north western Italy - analysis of two HYDROPTIMET test cases
}

\author{
D. Rabuffetti, M. Milelli, M. Graziadei, P. Mercogliano, and E. Oberto \\ ARPA Piemonte Area Previsione e Monitoraggio Ambientale C.so Unione Sovietica 216, 10134 Torino, Italy
}

Received: 24 October 2004 - Revised: 20 September 2005 - Accepted: 27 September 2005 - Published: 24 October 2005

\begin{abstract}
The HYDROPTIMET Project, Interreg IIIB EU program, is developed in the framework of the prediction and prevention of natural hazards related to severe hydrometeorological events and aims to the optimisation of HydroMeteorological warning systems by the experimentation of new tools (such as numerical models) to be used operationally for risk assessment. The object of the research are the Mesoscale weather phenomena and the response of watersheds with size ranging from $10^{2}$ to $10^{3} \mathrm{~km}^{2}$. Nonhydrostatic meteorological models are used to catch such phenomena at a regional level focusing on the Quantitative Precipitation Forecast (QPF). Furthermore hydrological Quantitative Discharge Forecast (QDF) are performed by the simulation of run-off generation and flood propagation in the main rivers of the interested territory. In this way observed data and QPF are used, in a real-time configuration, for one-way forcing of the hydrological model that works operationally connected to the Piemonte Region Alert System. The main hydro-meteorological events that interested Piemonte Region in the last years are studied, these are the HYDROPTIMET selected test cases of 14-18 November 2002 and 23-26 November 2002. The results obtained in terms of QPF and QDF offer a sound basis to evaluate the sensitivity of the whole hydro-meteorological chain to the uncertainties in the numerical simulations. Different configurations of non-hydrostatic meteorological models are also analysed.
\end{abstract}

\section{Introduction}

The HYDROPTIMET Project, approved in the framework of the international co-operation program Interreg IIIB MEDOCC, deals with the prevention of natural hazards and, in particular, those related to severe hydrometeorological events. The territory involved by the activities of the project

Correspondence to: M. Graziadei

(m.graziadei@arpa.piemonte.it) is well distributed along the Mediterranean coast and includes the regions of the western part of the Alps subject to severe events and to strong vulnerability due to the complex orography.

The Piemonte Region in the north-west of Italy is a predominantly alpine region covering $25000 \mathrm{~km}^{2}$. It is situated on the Padana plain and bounded on three sides by mountain chains covering $73 \%$ of its territory (Fig. 1). On the basis of historical data, available since the year 1800, the Piemonte Region is hit by calamitous meteorological events, on average, once every two years. The Authority of the Region decided to set up a specific organization for flood forecasting and damage mitigation from natural hazards. The SSRN (Natural Risks Evaluation Center) is the operational centre dedicated to the mitigation of the impact of hydrometeorological phenomena on the regional basis. It is a technical structure achieving two main tasks. (1) - Hydrometeorological survey: a group of technicians ensures that all the informative systems always run properly and all the data from the network are received. (2) - Hydro-meteorological forecast: groups of experts composed by Meteorologists, Hydrologist, Geologists and Snow scientist issue forecast and warning bulletins and develop studies and project to verify and improve the forecast and alert system. The key point of the regional alert system is the subdivision of the territory into homogeneous areas in terms of meteorological aspects and hydrological response during the occurrence of extreme events (see Fig. 2).

The main hydro-meteorological events that interested Piemonte Region in the last few years, 14-18 November 2002 and 23-26 November 2002, are analysed.

\section{Meteorological activities}

The goal of the meteorological activities at ARPA Piemonte is the study of different configurations of the Lokal Modell (LM) meteorological model, in order to optimise the QPF over the Piemonte warning areas. This variable is quite 


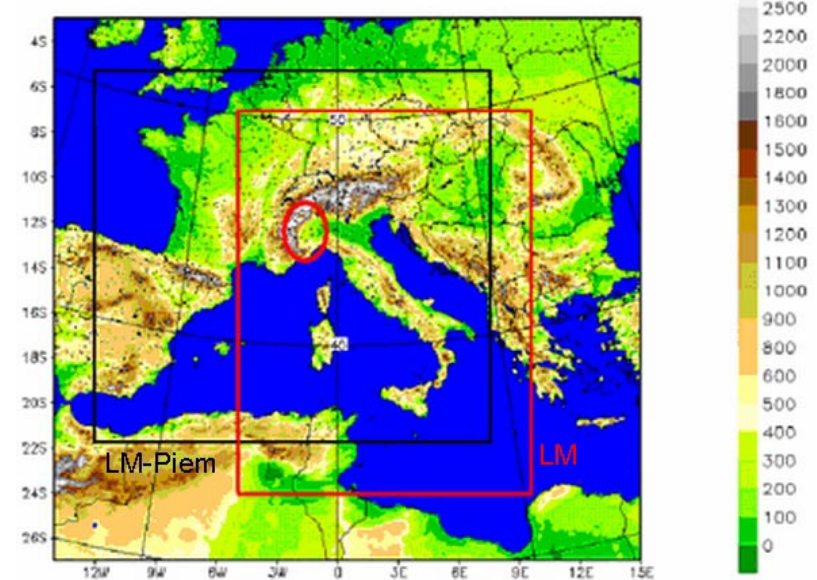

Fig. 1. Piemonte location circled with the evidence of the small LM and the large LM-Piem domains.

important since it is the input for the hydrological models uses to assess flood risk level.

The model used is the LM, non-hydrostatic limited area model developed in the framework of the COSMO project (COnsortium for Small-scale MOdelling). It is based on the primitive hydro-thermodynamical equations describing compressible non-hydrostatic flow in a moist atmosphere without any scale approximations. These kind of equations avoid restrictions on the spatial scales and on the domain size. The $7 \mathrm{~km}$ grid resolution is used. The simulations, hereafter indicated as LM-Piem, have been carried out on two events of intense precipitation that occurred in the North-West of Italy and, in particular, in Piemonte: first case between 14th and 16th of November 2002 (ITALIA1); second case between 24th and 26th of November 2002 (ITALIA2). For each event two $36 \mathrm{~h}$ forecast periods are selected: 14th November 2002 at 12:00 UTC (ITALIA1); 15 November 2002 at 12:00 UTC (ITALIA1); 24 November 2002 at 00:00 UTC (ITALIA2); 25 November 2002 at 00:00 UTC (ITALIA2). The simulations are performed enlarging the operational LM's domain in order to have the Piemonte region in the centre so avoiding boundary problems (Fig. 1). Moreover for LM-Piem initial and boundary conditions are taken from the ECMWF global model while LM's "father" is the global model GME. The analysis of the test cases shows that the global models can produce a clear divergence in the forecasted fields and this is reflected to the LM simulations as highlighted by the statistical indexes Threat Score (TS) and Bias (Murphy et al., 1987).

\section{Hydrological activities}

The aim is to provide flood forecasts using numerical hydrological models forced with QPF. This allows to analyse the sensitivity of hydrological forecasts to the QPF in reproducing the behaviour of the river network. The model used for this specific activity is the FloodWatch (DHI, Water and

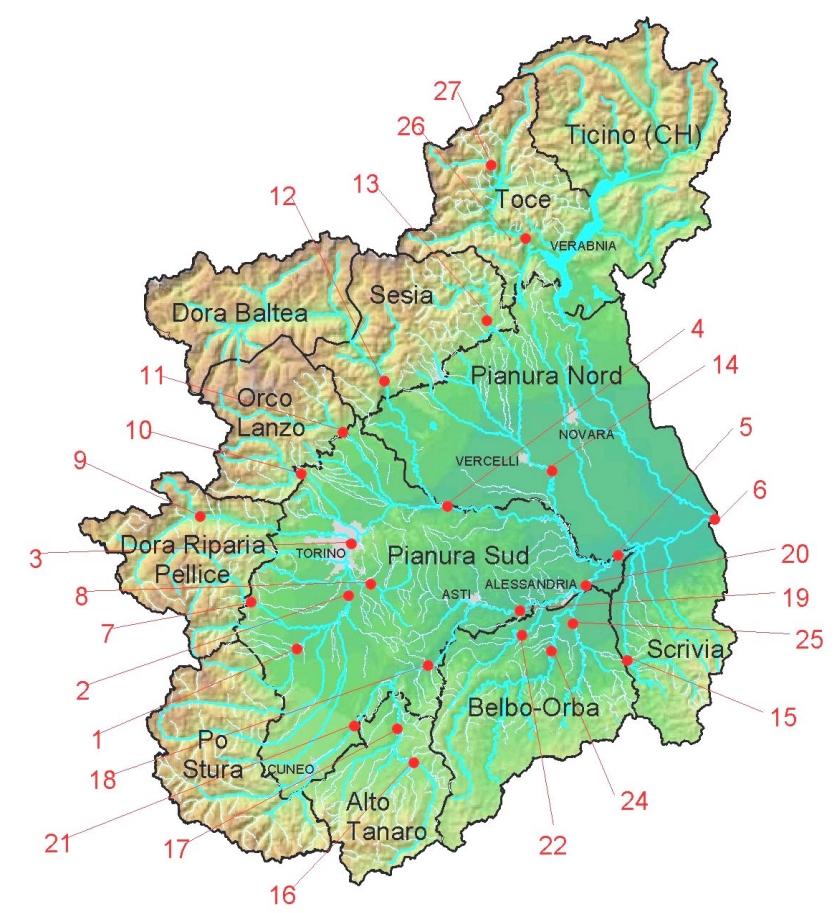

Fig. 2. Homogeneous alert areas, river network and location of the 27 cross sections considered.

Environment) developed for the Piemonte Region Alert System (Barbero et al., 2001). It consists in different modules for hydrological and river routing simulations. The rainfallrunoff module requires rainfall, potential evapo-transpiration and temperature as input. In order to carryout forecast simulations, estimates of rainfall and temperature input are required. Catchment runoff hydrographs are first computed, then these become the input for the hydrodynamic module. QPF estimated by the following different meteorological models are considered. The mean 6 hourly intensity is used for all the rainfall forecast.

- The global ECMWF and the local LM operational runs are the actual products available for exploiting in realtime hydrological forecasts.

- The "operational" model considers the meteorological forecasts issued for the Piemonte alert zones in terms of mean area rainfall. These values are interesting as they are daily used in the Italian hydrologic risk warning system. These are obtained by "ad hoc" evaluations by meteorologists expert on local weather and are based on different available information sources (meteo models, radar, real-time survey network).

- The LM-Piem configuration as explained in Sect. 2.

- "Perfect forecast" is based just on observed rainfall data and it is used to understand the hydrological model performance and to show how it is degraded by the use of meteorological estimates. 
Table 1. Synthesis of discharge forecast errors.

\begin{tabular}{lcccc}
\hline & \multicolumn{2}{c}{ Case study: ITALIA1 } & \multicolumn{2}{c}{ Case study: ITALIA2 } \\
\hline QPF Model & Mean Error & Standard Deviation & Mean Error & Standard Deviation \\
\hline Perfect forecast & -0.01 & 1.10 & 0.04 & 0.46 \\
operational & -0.14 & 1.03 & -0.03 & 0.57 \\
ECMWF & -0.31 & 1.15 & -0.28 & 0.41 \\
LM & -0.27 & 1.12 & -0.18 & 0.62 \\
LM-Piem & -0.29 & 1.26 & -0.16 & 0.44 \\
\hline
\end{tabular}
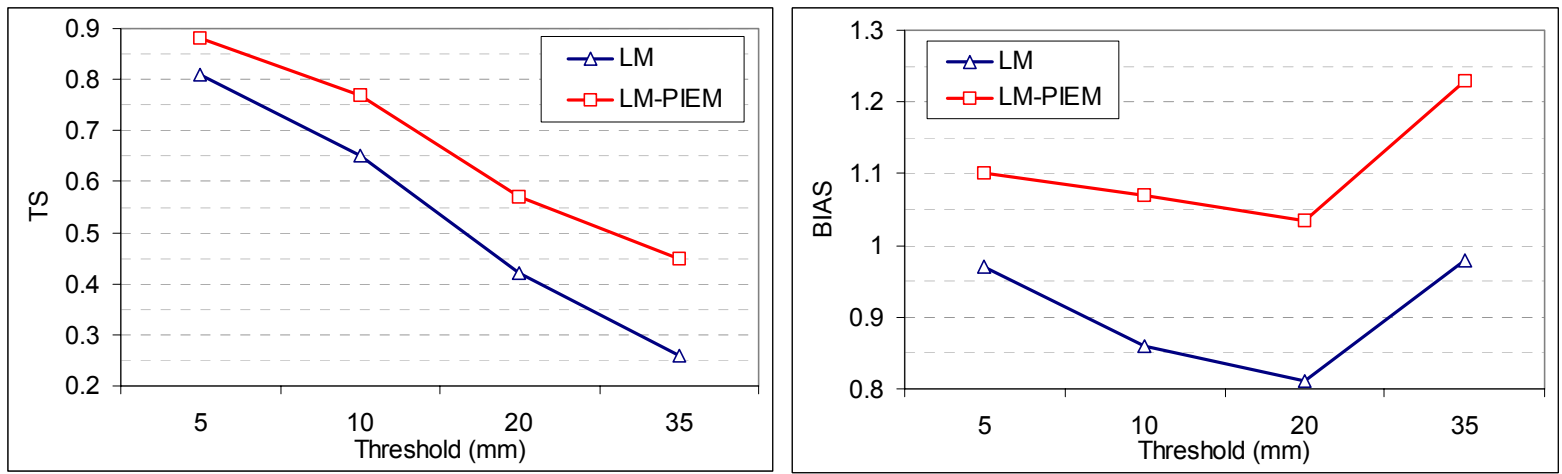

Fig. 3. Indexes of meteorological runs: TS (left) and BIAS (right).

To synthesize the hydrological model behaviour a relative error is calculated for each forecast (Eq. 1). Table 1 reports the mean error and the standard deviation for the test cases.

error $=\frac{\max \left(Q_{\text {forecast }}\right)-\max \left(Q_{\text {observed }}\right)}{\max \left(Q_{\text {observed }}\right)}$

QDF error is generally negative, meaning an underestimation of the hydrometeorological coupling. The "perfect forecast" shows the minimum mean error in both cases: this implies that generally the hydrological model itself has good performances; "Operational" chain has better performances than ECMWF, LM and LM-Piem probably because of the expert intervention. "ECMWF" has the highest mean error confirming the difficulties to force hydrological forecast on little and medium sized catchments with global model QPFs. Standard deviation values are quite similar for the different forecast systems except for LM-Piem in ITALIA1 and LM in ITALIA2 where some big overestimation occurred.

Furthermore, in Eq. (2), the normalized index, $i_{-} a$ is defined. This aims to evaluate how much the forecast errors depend on the advance of the forecast itself. To account for the geomorphologic differences among the studied basins, it's necessary to compare the forecast advance with the dynamics of each catchment, that can be represented by the lag time Tc.

$i_{\_} a=\frac{T(Q \max )-T O F}{T c}$

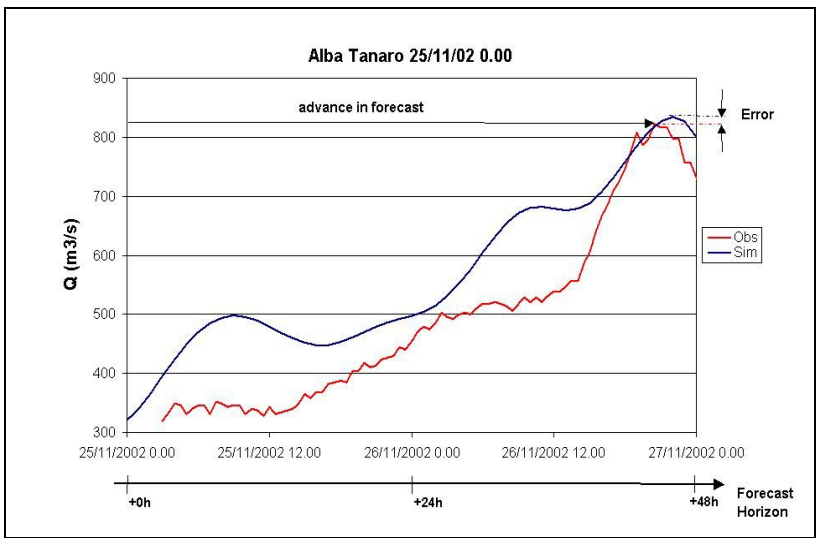

Fig. 4. Comparison between forecasted and observed hydrograph.

where $T(Q \max )$ is the time of the observed peak discharge, TOF is the time in which the forecast starts and their difference is the forecast advance with respect to peak.

An example is given in Fig. 4 for Alba cross section along Tanaro river using the operational model simulation with the run of 25/11/02 at 00:00.

Hydrological forecast errors versus the forecast advance is shown in Fig. 5. "Perfect forecast" errors cannot be plotted because in that case only observed data are used and so TOF has no meaning. The underestimation is not generally strictly linked to the advance of forecast highlighting the heavy role of the present uncertainties. 

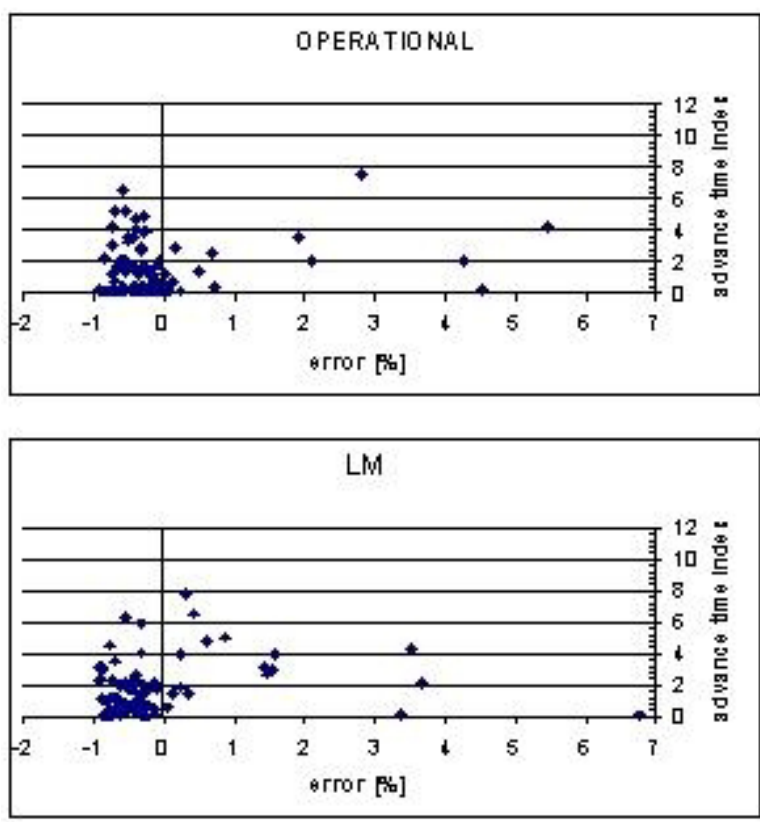

Fig. 5. QDF error versus advance time index.

Table 2. Warning system performance.

\begin{tabular}{llcccc}
\hline Case study & & \multicolumn{2}{c}{ ITALIA1 } & \multicolumn{2}{c}{ ITALIA2 } \\
\hline Model & TS & BIAS & TS & BIAS \\
\hline Perfect forecast & 0.15 & 0.15 & 0.33 & 0.33 \\
Operational & 0.46 & 0.46 & 0.00 & 0.00 \\
ECMWF & 0.00 & 0.00 & 0.00 & 0.00 \\
LM & 0.13 & 0.50 & 0.13 & 0.50 \\
LM-Piem & 0.08 & 0.08 & 0.25 & 0.25 \\
\hline
\end{tabular}

\section{The hydro-meteorological chain}

From an operational point of view, besides the error analysis in terms of discharge, it is very important to understand how these errors impact on the reliability of the alert system. The objective is to understand the relative weight of these sources of uncertainty in flood warning procedures reliability. False alarms and failed alarms need evaluating: for each cross section, the discharge simulations allow the definition of the expected risk level by the comparison of the expected flood peak and the discharge threshold. A contingency table can be created and the classical statistical indexes TS and BIAS calculated to point out the behaviour of the alert system during the test case events (Table 2).

The discharge thresholds are defined by means of off-line hydraulic analysis of each river reach and, when available considering historical flood data. The results obtained using the whole hydro-meteorological chain offer a sound basis to evaluate its sensitivity to the uncertainties in the numerical simulations related to the performance of alert system.
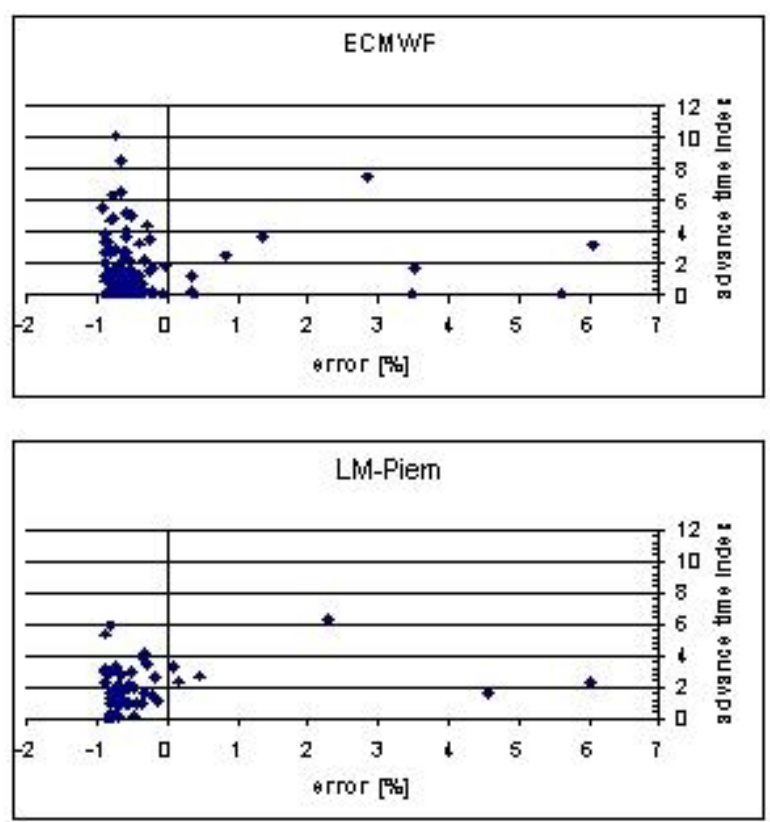

As one can note there is not a very good response. "Perfect forecast" results highlights the flood warning in little and medium sized catchments is far to be reliable even from the hydrological point of view. The "Operational" scheme can help mainly for long lasting events (ITALIA1) because during the events, expert meteorologists forecasts improve thanks to direct observations. LM models ensure better results than global ones do.

\section{Conclusion}

The results of meteorological activities prove that initial and boundary conditions are important sources of error in the forecast as far as very complex orography is involved. On the contrary, the dependency on the domain of integration is low, at least for these test cases. Globally runs derived from ECMWF better reproduce the rainfall fields.

It is confirmed that forcing hydrological forecast on little and medium sized catchments with global model QPFs produces significant errors. Furthermore, when considering the little and medium sized watersheds, the hydrologic model itself show important uncertainties due to the initial condition and to the simplified physical processes.

All the uncertainties present in the hydro-meteorological chain cause significant errors in terms of discharge forecasting. Of course, this impacts on the alert system performance but, at least for these test cases, the reliability of the whole warning system is not strictly related to the magnitude of those errors.

Future developments are addressed to improve the numerical models but need also to avoid deterministic forecasts take in account the use of probabilistic forecasts to face the residual uncertainty. 
Acknowledgement. This work was funded in the framework of HYDROPTIMET 2002-02-4.3-I-084 "Optimisation des outils de prévision Hydrométéorologique”, EU-INTERREG IIIB MEDOCC.

Edited by: L. Ferraris

Reviewed by: U. Parodi and another referee

\section{References}

Barbero, S., Rabuffetti, D., Wilson, G., and Buffo, M.: Development of a Physically-Based Flood Forecasting System: "MIKE FloodWatch" in the Piemonte Region, Italy, Proc. 4th DHI Software Conference, Helsingør, Denmark, 2001.

Murphy, A. H. and Winkler, R. L.: A general framework for forecast verification, Monthly Weather Review, 115, 1330-1338, 1997. 